\title{
VALORACIÓN ÉTICA DE LA CRANEOPLASTIA CON VENDAJE COMPRESIVO COMO FORMA DE LIMITACIÓN DE TRATAMIENTOS DE SOPORTE VITAL
}

\begin{abstract}
Alfonso Canabal Berlanga ${ }^{1}$, José Jara Rascón², Rafael Gómez Rodríguez ${ }^{1}$, Ricardo Abengózar Muela ${ }^{3}$, Francisco Javier Ruiz Hornillos ${ }^{4}$, José Manuel Moreno Villares ${ }^{5}$, Manuel de Santiago Corchado ${ }^{6}$, L. Fernando Viejo Llorente, José Carlos Abellán Salort ${ }^{6}$, Álvaro Gándara del Castillo ${ }^{8}$

Resumen: Este artículo analiza, desde una postura crítica, la utilización de la craneoplastia de compresión con vendaje como método de limitación de tratamiento de soporte vital (LTSV). Con esta técnica activa, algunos autores han propuesto provocar la muerte encefálica, posibilitando la donación de órganos. Al contrastar este procedimiento con las recomendaciones del documento de consenso sobre el tratamiento al final de la vida del paciente crítico, elaborado por el grupo de bioética de la SEMICYUC, se comprueba que los medios y fines de esta técnica no encajan con las actuaciones propias de la LTSV, que se basan en la retirada de medios de soporte vital o en su no inicio, al considerar dichos medios desproporcionados o extraordinarios en algunos casos, evitando así la obstinación terapéutica. La definición de LTSV permite clarificar los límites en los que, de un modo éticamente correcto y consensuado, las actuaciones al final de la vida se circunscriben a los fines de la medicina, evitando la sospecha de que dichas actuaciones puedan ser malinterpretadas como justificación para una obtención de órganos abusiva. El artículo concluye que la provocación directa de la muerte encefálica mediante la técnica de craneoplastia con vendaje no parece cumplir los criterios propios de la LTSV.
\end{abstract}

Palabras clave: craneoplastia, limitación de tratamiento de soporte vital, donación de órganos, craniectomía descompresiva

Ethical value of cranioplasty with oppressive binder as a way of limiting life support treatments

\begin{abstract}
This article analyzes, from a critical perspective, the use of cranioplasty with oppressive binder as a method to limit life support treatment (LLST). Some authors have proposed that this active technique provokes encephalic death, allowing organ donation. Contrasting this procedure with the recommendations of the consent document about treatment of critical patients at the end of life, elaborated by the bioethics group of SEMICYUC, it is shown that the means and ends of this technique do not match with the proper actions of LLST, based on the withdrawal of life support means or in not starting them, considering such means disproportionate or extraordinary in some cases, thus avoiding the therapeutic obstinacy. The definition of LLST allows to clarify the limits in which, in a way ethically fair and with a consensus, the acts at the end of life are included in the medical goals, avoiding the suspicion that these acts may be misinterpreted as justifying an abusive extraction of organs. This article concludes that the direct provocation of encephalic death by the technique of cranioplasty with binder does not appear to fulfill the criteria proper of LLST.
\end{abstract}

Key words: cranioplasty, limitation of life support treatment, organ donation, decompressive craniotomy

Valoraçáo ética da cranioplastia com curativo compressivo como forma de limitaçáo de tratamentos de suporte vital

Resumo: Este artigo analisa, a partir de uma postura crítica, a utilização da cranioplastia de compressão com curativo como método de limitação de tratamento de suporte vital (LTSV). Com esta técnica ativa, alguns autores têm proposto provocar a morte encefálica, possibilitando a doação de órgãos. Ao contrastar este procedimento com as recomendaçóes do documento de consenso sobre o tratamento do final de vida do paciente crítico, elaborado pelo grupo de bioética da SEMICYUC, se comprova que os meios e fins desta técnica não encaixam com as atuaçóes próprias da LTSV, que se baseiam na retirada de meios de suporte vital ou em seu não início, ao considerar os ditos meios desproporcionados ou extraordinários em alguns casos, evitando assim a obstinaçáo terapêutica. A definição de LTSV permite esclarecer os limites nos quais, de um modo eticamente correto e aceito, as atuaçóes ao final da vida se circunscrevem às finalidades da medicina, evitando a suspeita de que ditas atuações podem ser mal interpretadas como justificativa para uma obtenção de órgãos abusiva. O artigo conclui que a provocação direta da morte encefálica mediante a técnica da cranioplastia com curativo não parece cumprir os critérios próprios da LTSV.

Palavras-chave: cranioplastia, limitação de tratamento de suporte vital, doação de órgãos, craniectomia descompressiva

\footnotetext{
${ }^{1}$ Asociación de Bioética de Castilla la Mancha, España

Correspondencia: alcanabal@telefonica.net

${ }^{2}$ Asociación de Bioética de la Comunidad de Madrid, Espańa

${ }^{3}$ Departamento de Bioética de la Universidad Francisco de Vitoria de Madrid, Espańa

${ }^{4}$ Comité de Ética Asistencial de la Fundación Jiménez Díaz, Madrid, España

${ }^{5}$ Comité de Ética Asistencial del Hospital 12 de Octubre, Madrid, España

${ }^{6}$ Universidad Rey Juan Carlos, Madrid, España

${ }^{7}$ Comisión de Ética y Deontología del Colegio Médicos de Toledo, España

${ }^{8}$ Sociedad Española de Cuidados Paliativos (SECPAL), Espańa
} 


\section{Introducción}

La craneoplastia es el término que habitualmente se aplica a una intervención quirúrgica para la reconstrucción del defecto óseo de la bóveda craneal en pacientes previamente sometidos a una craniectomía descompresiva. Esta última es una intervención quirúrgica aplicada para reducir una hipertensión intracraneal refractaria a otras medidas terapéuticas $(1,2)$, generada por diferentes mecanismos fisiopatológicos, y típicamente por edema cerebral en una patología cerebral de diferente índole. Parece haber evidencias suficientes para defender su eficacia en disminuir la presión intracraneal(3-7), pero faltan ensayos clínicos de calidad que permitan definir claramente el efecto del tratamiento de descompresión mediante craniectomía a largo plazo. Parece que este procedimiento podría disminuir los días de ventilación mecánica y de estancia en cuidados críticos(8), pero sus beneficios a los seis meses son poco claros y contrastados con resultados funcionales controvertidos(9-12) en ocasiones con disfunción cerebral severa que a menudo generan dilemas éticos de difícil resolución(13-15). La craneoplastia, inversamente, sería una intervención reconstructiva para reponer hueso craneal o cubrir el defecto óseo con diferentes materiales sintéticos $(16,17)$ cuando ha cesado la causa que originó la hipertensión craneal y el pronóstico del paciente, por tanto, es favorable. Habitualmente se suele esperar un periodo de tiempo variable para realizarla, habitualmente de tres meses desde la realización de la primera intervención.

Recientemente, la polémica sobre la idoneidad de estos procedimientos se ha reabierto debido a que algunos autores, a partir de un único caso, han propugnado, como método éticamente correcto de Limitación de Tratamientos de Soporte Vital, el vendaje compresivo pericraneal (craneoplastia con vendaje)(18) para ser realizado en las unidades de enfermos críticos. Este nuevo procedimiento, que intenta aumentar la presión intracraneal hasta revertir lo logrado mediante craniectomía descompresiva, provoca de modo directo la muerte encefálica, y se aplicaría cuando los resultados neurológicos no fueren buenos y el paciente permaneciera en una situación de coma neurológico o daño cerebral profundo. Otra opinión publicada propone extender esta técnica tam- bién a los pacientes en estado vegetativo(19) que no presentan evolución a muerte encefálica. En ambos artículos subyace la justificación de que, mediante el vendaje craneal compresivo, estos pacientes puedan evolucionar a muerte encefálica y así poder ser donantes de órganos.

Con independencia del respeto que nos merecen sus autores, queremos ofrecer un punto de vista discrepante y extensamente argumentado, ya comunicado de forma preliminar(20), aclarando así los motivos por los que el vendaje craneal compresivo no puede ser una actuación correcta desde el punto de vista ético para lograr una limitación del tratamiento de soporte vital (LTSV).

\section{Desarrollo del tema}

\section{Donación de órganos y LTSV}

Queda claro que la principal motivación aducida para la craneoplastia con vendaje, según los autores que la propugnan, es la de conseguir una mayor disponibilidad de órganos. En el momento actual, se han puesto en práctica y se desarrollan en nuestro país, en los diferentes puntos geográficos, otros protocolos destinados a incrementar la oferta de órganos, como son los de donación en vivos y en asistolia, incluyendo el tipo Maastrich II y III, este último tras la $\operatorname{LTSV}(21)$. No entendemos por qué en un enfermo de similares características, si se pretende evitar la obstinación terapéutica por la situación clínica refractaria, no se realiza una LTSV, tal y como se describe en diferentes estudios y publicaciones(22-27). Con posterioridad a la aceptación de la retirada del soporte vital $-y$ de forma independiente a esta decisión - se puede ofrecer la oportunidad del tipo de donación tras la parada cardiorrespiratoria, procedimiento ya de por sí bastante cargado de dilemas éticos(28-32) y exigencias desde el punto de vista procedimental. Una de las dificultades presentes es que una decisión de LTSV debe ser totalmente independiente y con carácter previo al planteamiento de la donación de órganos, tal y como se señala claramente en el apartado de Aspectos éticos del documento de la Organización Nacional de Trasplantes (ONT) referenciado(22). En el supuesto que nos ocupa, es de la forma activa de limitar el esfuerzo terapéutico de la que derivará la consecuencia de la 
validez para la donación de órganos. El conflicto de intereses es difícilmente evitable y ambas decisiones se perciben como "difícilmente separables", puesto que la modalidad de limitación propuesta —el vendaje craneal compresivo- se presenta por sus defensores como una actuación encaminada específicamente a conseguir la posibilidad de donación. Por tanto, esta modalidad de LTSV, que en realidad parece más una reversión que una limitación del tratamiento previo, no parece seguir las recomendaciones de tratamiento al final de la vida del paciente crítico, desarrolladas por el Grupo de Bioética de la SEMICYUC(33).

Utilizar la craneoplastia con vendaje como forma de realizar la LTSV es aplicar un procedimiento activo. Aunque se quiere justificar como un modo de retirar o suprimir tratamientos desproporcionados, fútiles o extraordinarios, lo que se realiza es una maniobra que conlleva la muerte del paciente. Este procedimiento no se puede basar en el "principio del doble efecto", pues el objetivo perseguido por los defensores de la técnica es la muerte encefálica en el propio paciente, y como resultado directo la muerte del mismo y no la donación de órganos, cuyo beneficio indirecto redundaría además en otro individuo distinto. Por otra parte, dichos órganos se podrían obtener por diferentes vías, como puede ser la donación en asistolia. Por tanto, de partida, en estos postulados teóricos no se cumplen los criterios clásicos del principio aducido.

La única circunstancia que puede justificar una acción positiva que se siga de la muerte $-\mathrm{y}$ parcialmente influida por nuestra actuación - es en la sedación en la agonía(34-35), precisamente con base en el principio del doble efecto. El resto de actuaciones propias de la LTSV se basan en la retirada de medios de soporte vital o en el no inicio, al considerarse dichos medios desproporcionados o extraordinarios, evitando la obstinación terapéutica.

\section{Limitación del Esfuerzo Terapéutico}

En este supuesto de la craneoplastia, se considera fútil el tratamiento inicial, es decir, la craniectomía descompresiva. Pero esto no es así. Ciertamente los enfermos sometidos a una craniectomía descompresiva pueden abocar a una legítima decisión de LTSV. Pero tal decisión sigue estando determinada por el principio de beneficencia, de búsqueda del mejor bien del paciente o de evitación de cualquier mal. No ha perdido su condición de persona y su derecho a ser tratado como fin en sí mismo y no solo como medio. Si en función de los principios de la bioética se pretende evitar la obstinación terapéutica, que sería una actuación maleficente, lo lógico y más correcto desde el punto de vista ético es la retirada de los medios de soporte vital desproporcionados; pero la craniectomía no se puede retirar pues desencadenaría directamente la muerte encefálica, contrario al principio de no-maleficencia. De hecho, la craniectomía solo revierte el defecto de cráneo meses después, cuando se ha alcanzado un mínimo periodo de estabilización neurológica y los mecanismos de daño fisiopatológico neuronal han cesado. Si el enfermo tiene un daño cerebral severo, lo habitual es que se le mantenga de forma artificial con ventilación asistida y/o fármacos vasoactivos. En todo caso, la retirada de estas medidas se realizaría tal y como establecen las recomendaciones del Grupo de Trabajo de Bioética de la SEMICYUC, atendiendo al bienestar del enfermo y esperando que dicha supervivencia sin esperanza de curación finalice por medios naturales, con una muerte de tipo cardiorrespiratoria con los cuidados básicos y sedación paliativa del enfermo.

De los tres criterios determinantes de la futilidad (efectividad del tratamiento, beneficio del mismo evaluado por el paciente o por su familia y carga u onerosidad física, emocional, coste económico, etc. del mantenimiento del tratamiento) es obvio que la primera, la efectividad, es la más objetiva y cuantificable. Es el "bien médico" del enfermo. El que da luz y base para juzgar que una decisión clínica se atiene al bien del enfermo y a su dignidad como persona y fin en sí mismo, el que orienta al profesional de que el enfermo vulnerable está siendo tratado siempre en su beneficio y no en el de otros, cualesquiera sean. El que orienta una información veraz a la familia. Desde esta antropología clásica de la ética médica y la deontológica, pueden darse las condiciones exigibles: asegurar la futilidad de un tratamiento y, en tal caso, para la aplicación o no aplicación de una LTSV, que es entonces, en ambos casos, una decisión moral acertada. 
Por otra parte en el Código Deontológico médico actual, en el punto 3 del capítulo VII dedicado a la Atención médica al final de la vida(36), se dice que "el médico nunca provocará intencionadamente la muerte de ningún paciente, ni siquiera en caso de petición expresa por parte de éste", lo cual parece incompatible con la situación descrita. Tampoco parece poder sostenerla, con base en lo afirmado por el Código, el hecho de que un paciente hubiese solicitado tal cosa en el ejercicio de su autonomía y con carácter previo.

\section{Declaración UNESCO 2005}

La Declaración Universal sobre Bioética y Derechos Humanos de la UNESCO 2005(37), en su artículo 3 sobre la Dignidad humana y derechos humanos, señala: "Se habrán de respetar plenamente la dignidad humana, los derechos humanos y las libertades fundamentales. Los intereses y el bienestar de la persona deberían tener prioridad con respecto al interés exclusivo de la ciencia o la sociedad", lo que incluye la indisponibilidad de la vida humana, que no puede ponerse al servicio de otros intereses, por muy loables que parezcan, como la obtención de órganos, a través de actuaciones que facilitan la muerte del paciente. Quienes defienden la craneoplastia compresiva parecen malinterpretar el texto de la Declaración y sostienen lo contrario: que es lícito disponer de unas personas para lograr teóricos beneficios para otras.

La hipotética donación de órganos que se puede conseguir después, por útil que sea, no puede justificar un acto médico que persiga directamente la muerte del enfermo, salvo que se sostenga una ética meramente consecuencialista o utilitarista. Todos sabemos que la donación de órganos puede tener un enfoque predominante de este tipo $(38,39)$ y que incluso alguna legislación ha llegado a proponer que toda persona sea considerada como donante potencial, salvo que haya expresado su voluntad contraria a la donación(40). Pero todo ello es asumible solo cuando se garantice un respeto hacia la vida y la dignidad del futuro donante, gravemente enfermo o moribundo. En suma, existe una posibilidad de mejorar las indicaciones y reflexionar sobre la utilización de la craniectomía descompresiva, pero sin necesidad de, en función de otros antagónicos objetivos, se pretenda la desaparición de los beneficios que se han conseguido sobre las constantes del paciente.

Entre las opciones de realizar la craneoplastia con vendaje o de aplicar una LTSV para culminar en muerte por criterios cardiorrespiratorios, creemos que la segunda es la única opción que tiene un buen encaje bioético; ya que, aun así, no se impediría la donación de órganos, pues se podría realizar la donación en asistolia tipo Mastrich III, planteamiento que se debiera asumir de forma independiente del proceso de la donación y siempre de modo posterior a la primera decisión.

La justificación sobre la licitud ética del vendaje craneal compresivo se basa en la confusión de esta técnica con el concepto bien definido de limitación de soporte vital y, por otra parte, en un equívoco análisis principialista de la situación. En efecto, la limitación del esfuerzo terapéutico ha sido definida por la Sociedad Española de Cuidados Paliativos (SECPAL) como "la retirada o no inicio de medidas terapéuticas de soporte vital en pacientes en los que no están indicadas o son fútiles, permitiéndose así que la enfermedad de base siga su curso natural”. Esta definición no incluye, por tanto, la puesta en marcha de medidas activas conducentes a provocar directamente la muerte encefálica.

Por otra parte, cuando se dice que la realización de esta craneoplastia añade un innegable valor social, ya que facilita la donación de órganos para trasplante, se está olvidando que la provocación de la muerte no es un medio lícito para la obtención de órganos y que existen otras vías conducentes a facilitar la donación. Tampoco es un argumento inapelable el bien social, pues no es un concepto al que se le pueda atribuir un valor absoluto, salvo que se razone desde una visión utilitarista $(41,42)$ o propia del sociobiologicismo, en la cual se admite sacrificar el respeto hacia el individuo por el bien del grupo en su conjunto(43). Como afirma Pellegrino: "este abuso es un constante peligro en la bioética secular, donde la utilidad, y no la dignidad de la persona humana, es el criterio dominante" (44). Por alcanzar el interés social, aunque de extraordinaria importancia, no podemos ni debemos aceptar formas de proceder que no sean seguras desde el punto de vista de su corrección ética, incluyendo el respeto a la vulnerabilidad extrema de los pacientes con daño neurológico. 
Desde diferentes organizaciones y entidades de referente moral se considera una obligación de todos los profesionales sanitarios fomentar y promover la donación de órganos(36), pero ni el consuelo de los familiares ni los beneficios sociales deberían llevarnos a no respetar la dignidad y vulnerabilidad humanas (Declaración UNESCO, Art. 3), lo que dignifica la relación con nuestros pacientes y da valor a las personas, enfermas o moribundas, sin convertirlas en instrumentos de beneficio social.

El que con esta técnica no se vulneren los principios de beneficencia y no maleficencia es altamente discutible. En el momento de indicar la craniectomía descompresiva se establece esta indicación en beneficio del paciente y con la intención de mejorar sus constantes con el objetivo de disminuir la presión intracraneal. ¿Por qué después, cuando aún sigue siendo eficaz en cuanto al conseguir el fin para el que se aplicó, se plantea su reversión mediante la reposición de cráneo o materiales protésicos? Argumentando desde los clásicos principios de la bioética, en el momento en que se propone la craneoplastia compresiva lo que se busca es quitar los beneficios conseguidos, algo que parece argumentalmente un tanto incongruente, al menos utilizando el prisma del punto de partida. Lo que es beneficente y no maleficente en su aplicación, ahora que se sigue actuando de la misma manera resulta que no lo es. La respuesta lógica sería que no ha cambiado la potencial eficacia de la medida, sino la valoración global de la situación clínica, lo que haría deseable una LTSV. Si se tratara de retirar un medio de soporte vital se podría entender esa visión diferente, en diferentes momentos de la historia vital de la enfermedad, pero ciertamente la provocación de la muerte encefálica mediante una acción directa, del tipo de la analizada, no constituye una LSTV.

Dando a nuestras decisiones cobertura moral solo con el consentimiento informado de los familiares (autonomía por representación), podemos caer en un "hiperautonomismo" a ultranza, que se antepondría a las normas de buena práctica clínica y a nuestra responsabilidad como profesionales. La autonomía del paciente, habitualmente representada por los familiares, es de gran trascendencia, dado que la incorporación de las instrucciones previas no es mayoritaria, pero no podemos asumir que, en los casos objeto de este debate, interprete fácilmente la voluntad de los pacientes a nuestro cargo, ya que la magnitud y especificidad de la medida propuesta, de la craneoplastia, es difícilmente previsible, y la decisión de los familiares, en gran medida, puede verse influida por la información proporcionada por los profesionales sanitarios. Por ello, no deberíamos basar la corrección de nuestras actuaciones exclusivamente en la obtención de un consentimiento. La verdadera responsabilidad moral de nuestras acciones radica en la conciencia de cada profesional, como afirma Pellegrino. En la información inicialmente ofrecida para realizar la craniectomía descompresiva se explica a los familiares del paciente las bondades del método para disminuir la PIC, y tan solo unos días más tarde — según los defensores de esta técnica- se les habría de explicar que lo correcto es "deshacer" lo hecho, y llevar a cabo una intervención (vendaje craneal compresivo) orientada a que aumente nuevamente la PIC para que el paciente fallezca antes. Asumiendo la veracidad en la información en los dos casos, es fácil imaginar la confusión de la familia en la asimilación de esta información.

\section{Conclusiones}

La provocación directa de la muerte encefálica mediante la técnica de craneoplastia con vendaje no parece cumplir los criterios propios de la LTSV.

Debemos reflexionar sobre la moralidad de los cambios e innovaciones en la forma de proceder de nuestros actos médicos. No todo lo que se puede hacer se debe hacer sin más. Dada la importancia de la cuestión sobre las decisiones a tomar en pacientes con pronóstico de daño neurológico severo, probablemente se deba reflexionar más y buscar alternativas, que las hay. Ni podemos reducir todos nuestros dilemas al principialismo, hoy en considerable revisión, y simplificar los dilemas encuadrándolos forzadamente en unos principios cuando toda confrontación entre ellos está previamente decidida. Si queremos seguir mirando con orgullo y confianza nuestras actuaciones como profesionales y a nuestra organización de trasplantes, cuidémoslas para que nadie dude mínimamente de la corrección ética de los procedimientos para la obtención de órganos. 
Craneoplastia con vendaje compresivo como limitación de tratamientos de soporte vital - Alfonso Canabal Berlanga et al.

\section{Referencias}

1. Sauquillo J, Arikan F. Decompressive craniectomy for the treatment of refractory high intracraneal pressure in traumatic brain injury. Cochrane Database syst Rev 2006; 1 Cd003983 (update May 2008).

2. Cooper DJ, Rosenfeld JV, Murray L, Arabi YM, Davies AR, D’Urso P, Kossmann T, Ponsford J, Seppelt I, Reilly P, Wolfe R; DECRA Trial Investigators; Australian and New Zealand Intensive Care Society Clinical Trials Group. Decompressive craniectomy in diffuse traumatic brain injury. N Engl J Med 2011 Apr 21; 364(16): 1493-502. doi: 10.1056/ NEJMoa1102077. Epub 2011 Mar 25.

3. Amorim RLO, Teixeira MJ, Valbuza JS, De Oliveira MM, Panerai RB. Decompressive craniectomy: a meta-analysis of influences on intracranial pressure and cerebral perfusion pressure in the treatment of traumatic brain injury. J Neurosurg 2012; 117: 589-596.

4. Bor-Seng-Shu E, Figueiredo EG, Amorim RL, Teixeira MJ, Valbuza JS, de Oliveira MM, Panerai RB. Decompressive craniectomy: a meta-analysis of influences on intracranial pressure and cerebral perfusion pressure in the treatment of traumatic brain injury. J Neurosurg. 2012 Sep; 117(3): 589-596. doi: 10.3171/2012.6. JNS101400. Epub 2012 Jul 13.

5. Aarabi B, Hesdorffer DC, Ahn ES, Aresco C, Scalea TM, Eisenberg HM. Outcome following decompressive craniectomy for malignant swelling due to severe head injury. J Neurosurg 2006,104: 469-479.

6. Münch E, Horn P, Schürer L, Piepgras A, Paul T, Schmiedek P. Management of severe traumatic brain injury by decompressive craniectomy. Neurosurgery 2000; 47: 315-323.

7. Olivecrona M, Rodling-Wahlström M, Naredi S, Koskinen LO. Effective ICP reduction by decompressive craniectomy in patients with severe traumatic brain injury treated by an ICP-targeted therapy. J Neurotrauma 2007; $24: 927935$.

8. Madre H. Treatment interventions for severe traumatic brain injury: limited evidence, choice limitations. Journal of Medical Ethics 2012; 38(11): 662-663.

9. Cooper DJ, Rosenfeld JV, Murray L, Arabi YM, Davies AR, D’Urso P, et al. Decompressive Craniectomy in Diffuse Traumatic Brain Injury. N Engl J Med 2011; 364: 1493-1502.

10. Lubillo S, Blanco J, López P, Molina I, Domínguez J, Carrera L, et al. Papel de la craniectomía descompresiva en el enfermo neurocrítico. Med Intensiva 2009; 33: 74-83.

11. Hutchinson PJ, Corteen E, Czosnyka M, Mendelow AD, Menon DK, Mitchell P, et al. Decompressive craniectomy in traumatic brain injury: the randomized multicenter RESCUE study. Acta Neurochir Suppl. 2006; 96: 17-20

12. Pompucci A, De Bonis P, Pettorini B, Petrella G, Di Chirico A, Anile C. Decompresive craniectomy for traumatic brain injury: patient age and outcome. Neurotrauma 2007; 24: 1182-1188.

13. Honeybul S, Gillett G, Ho KM, et al. Ethical considerations for performing decompressive craniectomy as a life-saving intervention for severe traumatic brain injury. J Med Ethics. Published Online First, 17 July 2012. doi:10.1136/medethics-2012-100672.

14. Honeybul S, Gillett G, Ho H, Lind Ch. Ethical considerations for performing decompressive craniectomy as a lifesaving intervention for severe traumatic brain injury. J Med Ethics 2012; 38: 657-661.

15. Gillett GR, Honeybul S, Ho KM, Lind CRP. Neurotrauma and the RUB: where tragedy meets ethics and science. J Med Ethics 2010; 36(12): 727-730.

16. Sobani ZA, Shamim MS, Zafar SN, Qadeer M, Bilal N, Murtaza SG, Enam SA, Bari ME. Cranioplasty after decompressive craniectomy: An institutional audit and analysis of factors related to complications. Surg Neurol Int 2011; 2: 123.

17. Spetzger U, Vougioukas V, Schipper. Materials and techniques for osseous skull reconstruction. J. Minim Invasive Ther Allied Technol 2010 Apr; 19(2): 110-121.

18. Escudero D, Cofiño L, Gracia D, Palacios M, Casares M, Cabré L, Simón P, Miranda P, Martínez K, Iribarren S, Saralegui I, Simó RM, de León B y Español V. Craneoplastia con vendaje. nuevas formas de limitación del tratamiento de soporte vital y donación de órganos. Med Intensiva 2013; 37: 180-184.

19. Rivero-Garvia M, Mayorga-Buiza MJ, Rodríguez-Boto G, Aldabó-Pallas T, Márquez Rivas J. Vendaje compresivo en pacientes en estado vegetativo. Med Intensiva 2013 Oct; 37(7): 500-501.

20. Canabal Berlanga A. Discrepancia a la utilización de la craneoplastia con vendaje como forma de limitación de tratamientos de soporte vital. Otro punto de vista. Medicina Intensiva. 2014; http://dx.doi.org/10.1016/j.medin.2013.12.009 [en prensa].

21. Solís García del Pozo J, Gómez Pérez I. El límite del esfuerzo terapéutico en las publicaciones médicas españolas. Cuad. Bioét 2012; XXIII, 3a: 641-656.

22. Organización Nacional de Trasplantes. Donación en Asistolia en España: Situación Actual y Recomendaciones. Documento de Consenso Nacional 2012. 
23. Esteban A, Gordo F, Solsona L, Alía I, Caballero J, Bouza C, et al. Withdrawing and withholding life support in the intensive care unit: a Spanish prospective multi-centre observational study. Intensive Care Medicine 2001; 27(11): 1744-1749.

24. Sprung CL, Cohen SL, Sjokvist P, Baras M, Bulow HH, Hovilehto S, Ledoux D, Lippert A, Maia P, Phelan D, Schobersberger W, Wennberg E, Woodcock T. Ethicus Study Group. End-of-life practices in European intensive care units: the Ethicus Study. JAMA 2003; 290(6): 790-797.

25. Angus DC, Barnato AE, Linde-Zwirble WT, Weissfeld LA, Watson RS, Rickert T, Rubenfeld GD; Robert Wood Johnson Foundation ICU End-of-Life Peer Group. Use of intensive care at the end of life in the United States: An epidemiologic study. Crit Care Med 2004; 32(3): 638-643.

26. Poyo-Guerreroa R, Cruz A, Laguna M, Mata J; Comité de Ética del Hospital Son Llatzer de Palma de Mallorca (España). Experiencia preliminar en la introducción de la limitación de terapias de soporte vital en la historia clínica electrónica. Med Intensiva 2012; 36(1): 32-36.

27. Ferrand E, Robert R, Ingrand P, Lemaire F; French LATAREA Group. Withholding and withdrawal of life support in intensive-care units in France: a prospective survey. French LATAREA Group. Lancet 2001; 357(9249): 9-14.

28. Vincent JL, Brimioulle S. Non-Heart-Beating Donation: Ethical Aspects. Transplantation Proceedings 2009; 41: 576578.

29. Bos MA. Ethical and legal issues in non-heart-beating organ donation. Transplantation 2005; 79(9): 1143-1147.

30. Zeiler K, Furberg E, Tufveson G, Welin S. The ethics of non-heart-beating donation: how new technology can change the ethical landscape. J Med Ethics 2008; 34(7): 526-529.

31. Motta ED. The ethics of heparin administration to the potential non-heart-beating organ donor. J Prof Nurs 2005; 21(2): 97-102.

32. Aulisio MP, Devita M, Luebke D. Taking values seriously: Ethical challenges in organ donation and transplantation for critical care professionals. Crit Care Med 2007; 35 (2 Suppl): S95-101.

33. Monzón Marín JL, Saralegui Reta I, Abizanda i Campos R, Cabré Pericas L, Iribarren Diarasarri S, Martín Delgado MC, Martínez Urionabarrenetxea K, Grupo de Bioética de la SEMICYUC. Recomendaciones de tratamiento al final de la vida del paciente crítico. Med Intensiva 2008; 32(3): 121-133.

34. Cuervo Pinna MA, Sánchez Correas MA, Sánchez Posadas R, Alonso Prado ME, Ruiz Márquez MP, Espinosa Rojas JA, et al. (Grupo de trabajo de Tratamientos del Programa Regional de Cuidados Paliativos del Servicio Extremeño de Salud). Sedación en cuidados paliativos. Disponible en http://www.secpal.com/guiasm/index.php?acc=see_guia\&id_ guia $=8$ (Consultado el 14 de enero de 2014).

35. Organización Médica Colegial (OMC) y Sociedad Española de Cuidados Paliativos (SECPAL). Guía de sedación paliativa. Disponible en https://www.cgcom.es/sites/default/files/guia_sedaccion_paliativa_0.pdf (Consultado el 13 de enero de 2014).

36. Consejo General de Colegios Oficiales de Médicos. Código de Deontología Médica. Guía de Ética Médica. Julio 2011. Disponible en https://www.cgcom.es/sites/default/files/codigo_deontologia_medica.pdf (Consultado el 21 de diciembre de 2013).

37. Organización de las Naciones Unidas para la Educación, la Ciencia y la Cultura. Declaración Universal sobre Bioética y Derechos Humanos. 19 de octubre de 2005.

38. García Marcos F. Aspectos éticos en trasplante de órganos. Cuadernos de Bioética 2001; 12(45): 253-265.

39. López de la Vieja MT, Velayos C. Educación en Bioética: Donación y trasplante de órganos. Salamanca, Aquilafuente: Ediciones Universidad de Salamanca; 2008.

40. Ley 30/1979, de 27 de octubre, sobre extracción y trasplante de órganos. BOE, 6 de noviembre de 1979; n 266: 25742 -25743 .

41. Bentham J. Introducción a los principios de la moral y de la legislación (1789). En: Bentham J. Antología. Edición de Josep Ma Colomer. Barcelona: Ediciones Península; 1991.

42. Smart JJ, Williams B, (eds.) Utilitarianism For and Against. Cambridge: Cambridge University Press, 1973.

43. Wilson EO. Sociobiology: the new synthesis. Harvard University Press; 1975.

44. Pellegrino, E.D. The Lived Experience of Human Dignity. In Human Dignity and Bioethics. Washington DC.: President's Council on Bioethics; 2008. 